\title{
Strength of conditioning determines the effects of septo-hippocampal lesions on taste aversion learning
}

\author{
ARTHUR J. NONNEMAN and STEPHEN D. CURTIS \\ University of Kentucky, Lexington, Kentucky 40506
}

\begin{abstract}
Several authors have argued that the formation of conditioned flavor aversions (bait-shyness) is qualitatively different than the formation of associations between exteroceptive cues and external noxious reinforcers. Furthermore, it is argued that these two presumably distinct forms of learning are dependent on different neural substrates. This contention is strengthened by several recent reports that lesions of the septum or hippocampus that disrupt locomotor passive avoidance learning do not impair baitshyness, even though taste aversion studies typically use a passive avoidance paradigm. Nevertheless, other studies have reported significant taste aversion deficits after septal or hippocampal lesions. In the present study, we suggest that the primary factor that determines whether septo-hippocampal lesions impair taste aversion learning is the strength of the conditioning method employed. Experiment 1 showed that large lesions of the septum, the fimbria-fornix, or the dorsal hippocampus did not impair a flavor aversion learned with a strong conditioning paradigm. In contrast, both septal and hippocampal lesions disrupted a flavor aversion learned with the weaker conditioning method of Experiment 2. We conclude that it is not yet possible to state that the association of flavor cues with interoceptive events depends on neural circuits different from those involved in the association of exteroceptive cues and events.
\end{abstract}

In recent years, a great deal of attention has been directed toward the study of "biological constraints" on learning in various species (Hinde, 1973; Seligman \& Hager, 1972). One example of such a presumed biological constraint is the phenomenon of baitshyness or taste aversion learning. Baitshyness consists of a rapid association between distinctive flavors (or odors) and subsequent illness with a resultant avoidance of such flavors or odors on later encounters. Strong taste aversions can often be demonstrated after a single CS (taste)-US (illness) pairing and even with interstimulus delays of several hours (Rozin \& Kalat, 1971). Moreover, this association seems to be quite specific. Taste cues are readily associated with internal malaise but not with footshock; conversely, exteroceptive stimuli such as lights or tones are readily associated with shock but not with internal malaise (Garcia \& Koelling, 1966). Such effects have led to the suggestion that taste aversion learning does not follow the same "laws" of association as conditioning between exteroceptive

This research was supported by USPHS Grant MH 25649 to A. J. Nonneman. We thank J. V. Corwin for helpful comments on a preliminary draft of this manuscript. Portions of this study were presented at the Animal Behavior Society meetings, Wilmington, North Carolina, May 24, 1975. Address reprint requests to Arthur J. Nonneman, Department of Psychology, 104 Kastle Hall, University of Kentucky, Lexington, Kentucky 40506. cues and external noxious reinforcers (Rozin \& Kalat, 1971). Furthermore, these apparently different kinds of learning may be dependent on different neural substrates (Garcia \& Koelling, 1966). If this is true, it should be possible to differentially disrupt avoidance conditioning based on exteroceptive or interoceptive reinforcers by lesioning the appropriate regions of the brain.

Most of the research that has attempted to determine whether separate neural substrates underlie the association of interoceptive and exteroceptive cues and reinforcers has examined the effect of septal or hippocampal lesions on baitshyness and locomotor passive avoidance. These studies were guided by the knowledge that damage to either area produces severe deficits on locomotor passive avoidance, and that the baitshyness situation follows a passive avoidance paradigm.

Several of these studies have reported a partial dissociation between taste aversion and passive avoidance acquisition in rats with septal or hippocampal lesions (Hobbs, Elkins, \& Peacock, 1974; McGowan, Garcia, Ervin, \& Schwartz, 1969; McGowan, Hankins, \& Garcia, 1972). In these studies, locomotor passive avoidance was severely disrupted, but taste aversion learning was unaffected by the lesions. However, other studies have reported deficits either in the acquisition or extinction of a conditioned taste aversion after lesions of the septum (Kolb, Nonneman, \& Abplanalp, 1977) or the hippo- 
campus (Best \& Orr, 1973; Krane, Sinnamon, \& Thomas, 1976; McFarland, Kostas, \& Drew, in press; Miller, Elkins, Fraser, Peacock, \& Hobbs, 1975; Miller, Elkins, \& Peacock, 1971). These studies differ in a number of respects, including size and location of the lesion within the septo-hippocampal system, the nature of the CS, the nature and route of administration of the US, presence or absence of preconditioning exposure to the $\mathrm{CS}$, the dependent measures employed, and a number of other procedural variables. Considering these multiple differences, it should perhaps come as no surprise that apparently contradictory results have been obtained. This study represents an attempt to determine the source of these contradictions.

Two primary variables are examined: size and location of the lesion within the septo-hippocampal system and strength of conditioning. The results of this study, together with a review of the extant literature, suggest that septal or hippocampal lesions disrupt taste aversion learning in rats under the same conditions in which they disrupt avoidance conditioning produced by the pairing of exteroceptive cues and reinforcers.

\section{EXPERIMENT 1}

Best and Orr (1973) have suggested that the extent of direct or indirect destruction of the fimbria-fornix fiber system is a major determinant of the results in taste aversion studies with septal or hippocampal lesions. In their study, lesions of the posterolateral hippocampus had little effect on the acquisition of a conditioned taste aversion, whereas dorsal hippocampal lesions that invaded the fimbria produced a dramatic deficit. Because of the fimbrial damage, which interrupted a major efferent pathway of the hippocampus, the dorsal lesions were functionally larger than the posterolateral lesions. This interpretation is consistent with the results of a number of other studies as well. In general, those studies reporting large hippocampal lesions that include the fimbria (Krane et al., 1976; Miller et al., 1971) report a stronger disruption of conditioned taste aversion than do those studies with lesions that largely spare the fimbria (Kolb et al., 1977; McGowan et al., 1972; Murphy \& Brown, 1974; Thomka \& Brown, 1975). In like manner, those studies using restricted septal lesions that largely spared the fimbria-fornix (Hobbs et al., 1974; McGowan et al., 1969; McGowan et al., 1972) found no taste aversion deficit, whereas Kolb et al. (1977) reported a significant impairment after large septal lesions that included the columns of the fornix, the dorsal fornix, and in many cases the hippocampal commissure.

The present experiment directly tested the notion that the fimbria-fornix plays a critical role in the formation of conditioned taste aversions. Separate groups of animals received lesions of the dorsal hippocampus, the septum, the fornix and hippocampal commissure, or sham operations. A strong conditioning paradigm was used.

\section{Method}

Subjects. The subjects were 27 male Sprague-Dawley albino rats aged approximately 120 days at the time of surgery. They were housed individually in wire-mesh suspended cages and maintained on a $12 \mathrm{~h}$ light on, $12 \mathrm{~h}$ light off schedule. Teklad rat pellets were available ad lib throughout the experiment.

Surgery. Surgery was performed under antiseptic conditions, and all rats received a single 50,000-unit injection of penicillin at the end of surgery. The rats were anesthetized with sodium pentobarbital $(50 \mathrm{mg} / \mathrm{kg})$, the scalp was incised on the midline, and the periosteal fascia was scraped to the side. For shamoperated controls, the incision was then closed with wound clips. All other subjects received electrolytic lesions produced by anodal dc current passed between an anal cathode and a stainless steel electrode insulated with enamel except for approximately $.5 \mathrm{~mm}$ at the tip. The coordinates for stereotaxic placement, determined from the atlas by Skinner (1971), and the current parameters for each lesion groups were as follows: fornix, AP $-0.5 \mathrm{~mm}, \mathrm{~L} 0.5 \mathrm{~mm}, \mathrm{~V} 4.0 \mathrm{~mm}, 2.0 \mathrm{~mA}$ for $20 \mathrm{sec}$; septum, AP +1.0 mm, L $0.5 \mathrm{~mm}, \mathrm{~V} 4.0 \mathrm{~mm}, 2.0 \mathrm{~mA}$ for $20 \mathrm{sec}$; dorsal hippocampus, AP $-3.0 \mathrm{~mm}$, L $2.0 \mathrm{~mm}, \mathrm{~V} 3.5 \mathrm{~mm}, 3.0 \mathrm{~mA}$ for $30 \mathrm{sec}$. Depth coordinates were measured from the skull. A 10-day recovery period separated surgery and testing.

Procedure. The procedure was essentially the same as that reported by Kolb et al. (1977). The animals were adapted to a $231 / 2$-h water-deprivation schedule for 1 week prior to testing. The rats remained in their home cages throughout the testing. Each daily $1 / 2-h$ test session began when a bottle containing a premeasured volume of fluid was placed on the front of the cage. After $15 \mathrm{~min}$, this bottle was removed from the cage, the amount of fluid consumed was recorded, and another bottle containing tap water was placed on the cage for $15 \mathrm{~min}$. On Days 5 , 8 , and 10 , the first bottle contained .12-M LiCl, which served both as the $\mathrm{CS}$ and the US. Consumption of $\mathrm{LiCl}$ on Day 5 provided a measure of neophobia, and $\mathrm{LiCl}$ consumption on Days 8 and 10 provided the measures of taste aversion.

At the completion of this experiment, the rats were tested for several weeks on tests of social behavior and emotionality before being sacrificed with an overdose of sodium pentobarbital and intracardially perfused with normal saline followed by $10 \%$ Formalin in saline. The brains were then removed from the skulls, fixed for several additional days in Formalin, cut on a freezing microtome at $40 \mu$, mounted on slides, and stained with cresyl violet.

Statistics. Since there was considerable variability in baseline water consumption between subjects within each group, suppression ratios were used for all statistical analyses of neophobia and taste aversion. These ratios took the form: Day X consumption/Day X plus baseline consumption. Thus, a ratio of 0.5 would indicate no change from baseline, whereas a ratio smaller than 0.5 would indicate suppressed consumption, and a larger ratio would indicate increased consumption over baseline water intake. All statistical analyses used two-tailed t tests.

\section{Results}

Anatomical. The extent of the lesions is shown in Figure 1, which presents the largest and smallest lesion in each group. The septal lesions destroyed the medial and lateral septal nuclei in all animals and extended into the most rostral portion of the body of the fornix in several animals. The fornix lesions 

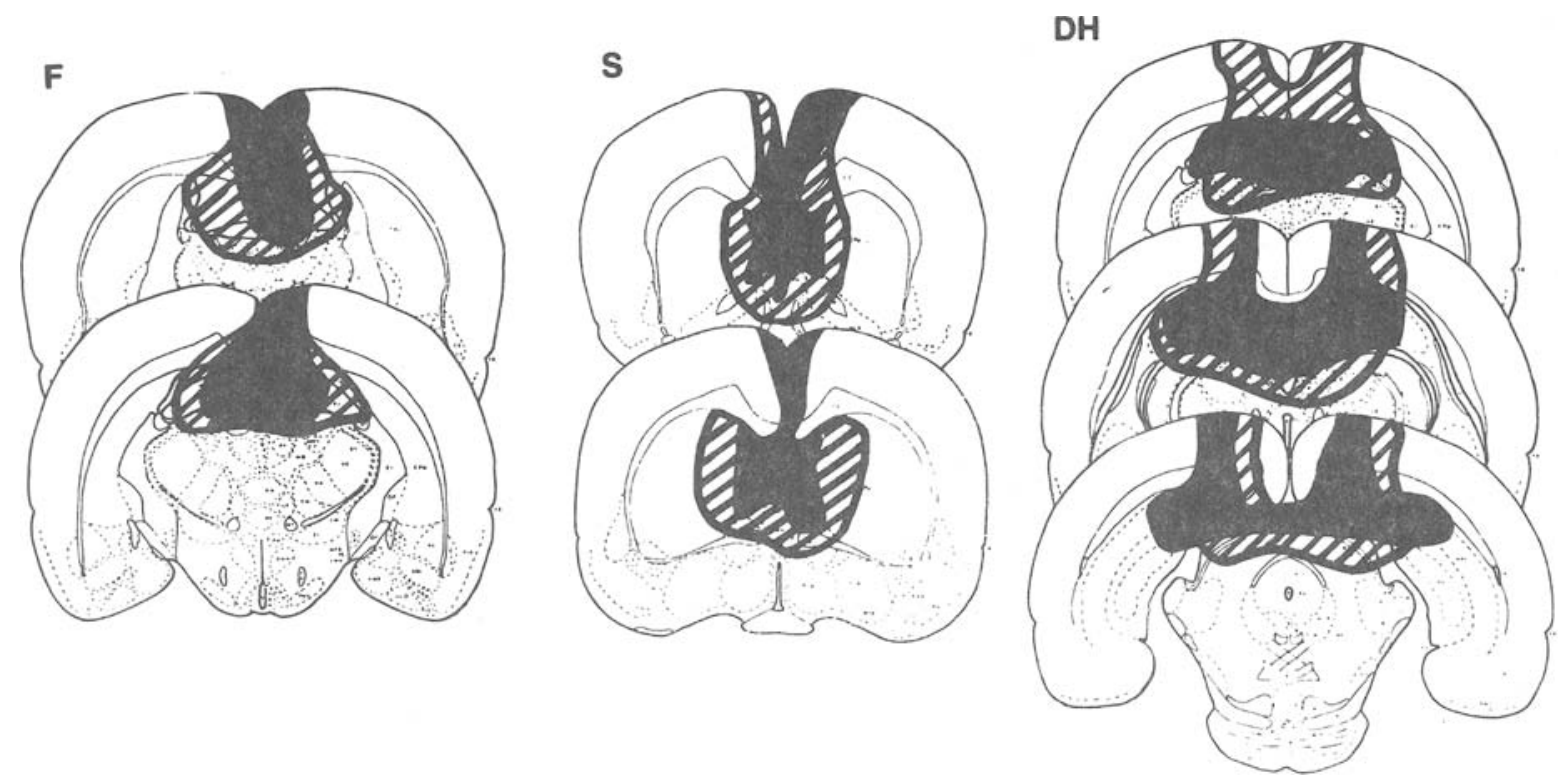

Figure 1. Reconstructions of the smallest (black) and largest (diagonal lines) lesions for each lesion group of Experiment 1. $\mathrm{F}=$ fornix, $\mathrm{S}=$ septum, $\mathrm{DH}=$ dorsal hippocampus.

destroyed the medial portion of the body of the fornix in all subjects. In the three rats with the largest lesions, the entire body of the fornix, the caudal septum, and the hippocampal commissure were destroyed. The hippocampal lesions destroyed the antero-dorsal hippocampus in all subjects and variable amounts of the hippocampal commissure, dorsal thalamus, and midbrain in different subjects. There was no significant correlation between any of the behavioral measures and amount of damage to any of these structures.

Behavioral. The behavioral results are summarized in Table 1. There were no significant differences between groups on mean baseline water consumption during Days 1-4 (t tests, $p>.05$ ). All groups showed a significant suppression of fluid intake (neophobia) on Day 5 ( $t$ tests, $p<.05$ ), and there were no significant differences between groups. On Days 8 and 10, all groups showed essentially complete suppression of $\mathrm{LiCl}$ intake (taste aversion) and there were no differences between groups.

\section{Discussion}

These results are consistent with a number of studies (Best \& Orr, 1973; Hobbs et al., 1974; Kolb et al., 1977; McGowan et al., 1969; McGowan et al., 1972; Miller et al., 1975; Murphy \& Brown, 1974; Thomka \& Brown, 1975) that have shown that an intact septo-hippocampal system is not essential for taste aversion learning. In each of these earlier studies, strong conditioned taste aversions were demonstrated in rats with partial, though sometimes extensive, destruction of the hippocampus or the septum. The present experiment extends these findings by showing that efficient taste aversion learning can occur even after total disconnection of the septum and hippocampus produced by complete lesions of the fornix at the level of the hippocampal commissure. Thus, the hypothesis that extent of damage to septohippocampal connections might be the primary determinant of deficits in taste aversion learning after hippocampal lesions was not confirmed.

There are a number of other potentially important differences between those studies that have reported deficits in conditioned taste aversions after hippocampal lesions and those that report no lesion effect. Perhaps the most striking of these differences is the nature of the UCS employed. For example, four of the five studies that reported no impairment of taste aversion acquisition after large hippocampal lesions

Table 1

Mean Volume (in Milliliters) of Fluid Consumed in 15-min Period by Rats with Lesions to Septo-Hippocampal System

\begin{tabular}{|c|c|c|c|c|c|c|c|c|c|}
\hline \multirow[b]{2}{*}{ Group } & \multirow[b]{2}{*}{$\mathbf{N}$} & \multicolumn{2}{|c|}{ Days $1-4\left(\mathrm{H}_{2} \mathrm{O}\right)$} & \multicolumn{2}{|c|}{ Day $5(\mathrm{LiCl})$} & \multicolumn{2}{|c|}{ Day $8(\mathrm{LiCl})$} & \multicolumn{2}{|c|}{ Day $10(\mathrm{LiCl})$} \\
\hline & & Mean & Range & Mean & Range & Mean & Range & Mean & Range \\
\hline Control & 7 & 15.0 & $12.9-19.5$ & 10.2 & $4-20$ & 1.8 & $1-2.5$ & 2.4 & $1-3.5$ \\
\hline Septal & 7 & 14.4 & $9.3-17.8$ & 10.2 & $9-12$ & 1.9 & $1-3$ & 1.6 & $1-3$ \\
\hline Fornix & 6 & 13.4 & $11.0-19.5$ & 6.2 & $4-11.5$ & 1.8 & $1-2$ & 2.4 & $2-4$ \\
\hline Hippocampal & 7 & 14.1 & $10.7-16.1$ & 9.1 & $5-15$ & 1.5 & $1-2$ & 2.4 & $1.5-4$ \\
\hline
\end{tabular}


(Kolb et al., 1977; Murphy \& Brown, 1974; Thomka \& Brown, 1975; present study) used $\mathrm{LiCl}$ as the US; the fifth (Miller et al., 1975) used cyclophosphamide. On the other hand, two of the three studies reporting an acquisition deficit after hippocampal lesions (Best \& Orr, 1973; Krane et al., 1976) used apomorphine as the US; the third (Miller et al., 1971) used X-ray.

Strength of conditioning is an important factor in determining the apparent effects of CNS damage on baitshyness, and the level of conditioning supported by different drugs is known to differ. For example, Revusky and Gorry (1973) reported that lithium supports stronger taste aversion conditioning than apomorphine, while emetine supports an intermediate level of conditioning in both rats and monkeys. Experiment 1 used a particularly strong conditioning paradigm. $\mathrm{LiCl}$ served as the US, and the rats drank approximately twice as much $\mathrm{LiCl}$ as Revusky and Gorry's highest dose, the dose typically used in taste aversion studies. Furthermore, the rats were not preexposed to the CS (cf. McFarland et al., in press), and the self-administration situation guaranteed minimal temporal separation between the taste of the novel $\mathrm{LiCl} \mathrm{CS}$ and the $\mathrm{LiCl}$-induced internal malaise.

\section{EXPERIMENT 2}

The results of Experiment 1 suggested that the effects of septo-hippocampal lesions on baitshyness might depend on the strength of conditioning obtained. Best and Orr (1973) and Miller et al. (1971) had previously reported that hippocampal lesions disrupt taste aversions learned under weak conditioning paradigms that paired a preferred saccharin CS and an apomorphine or X-ray US. At the time this research was conducted, there were no such reports in which $\mathrm{LiCl}$ had served as the US, although, in one study that preceded this one, Kolb et al. (1977) found a taste aversion deficit in rats with septal lesions when $\mathrm{LiCl}$ served both as the US and CS. ${ }^{1}$ This result suggested that under appropriate conditions, septo-hippocampal lesions might disrupt taste aversions supported by $\mathrm{LiCl}$. The second experiment tested this notion by pairing a preferred sucrose CS with a reduced dose of $\mathrm{LiCl}$ as the US.

\section{Method}

Subjects. The subjects were 28 male Sprague-Dawley albino rats aged 120 days at the start of this experiment. They were randomly assigned to three groups: septal lesions $(\mathrm{N}=8)$, total hippocampal lesion $(\mathrm{N}=8)$, and control $(\mathrm{N}=12)$. One septal operate died before the end of testing and one control was excluded from data analysis because its bottle broke during the baitshyness test on Day 7. For septal operates, the stereotaxic coordinates and current parameters were the same as those used to produce septal lesions in Experiment 1. For hippocampal operates, coordinates and current parameters were AP $-3.0 \mathrm{~mm}, \mathrm{~L} 1.5 \mathrm{~mm}$, V $3.5 \mathrm{~mm}$; AP $-4.0 \mathrm{~mm}$, L 2.0 and $3.0 \mathrm{~mm}, \mathrm{~V} 3.5$ and $5.5 \mathrm{~mm}$, respectively; AP $-5.0 \mathrm{~mm}$, L $5.0 \mathrm{~mm}, \mathrm{~V} 5.5 \mathrm{~mm}, 3.0 \mathrm{~mA}$ for $20 \mathrm{sec}$ at each placement. This represented an effort to destroy the entire hippocampus bilaterally. Surgery, sacrifice of the animals, and histological processing of the brains were identical to Experiment 1.

Procedure. The procedure was identical to that of Experiment 1 with the following exceptions: Sucrose $(15 \% \mathrm{w} / \mathrm{v})$ was the CS. The US $(2 \mathrm{ml} / \mathrm{kg}$ of $.3-\mathrm{M} \mathrm{LiCl})$ was injected IP 15 min after the completion of sucrose drinking. There were only 3 days of baseline water consumption measures prior to the initial sucrose presentation on Day 4. There was only one measurement of taste aversion to sucrose on Day 7. A recovery period of 2 weeks separated surgery and testing.

\section{Results}

The results are presented in Table 2. Every group showed a significant increase in fluid consumption when first given access to sucrose on Day 4 (t tests, $\mathrm{p}<.05$ ), and the groups did not differ from one another on initial (Days 1-3) water consumption or on initial (Day 4) sucrose consumption (t tests, $p>.05$ ). On the baitshyness test (Day 7), all groups showed a significant decrease in sucrose consumption (t tests, $\mathrm{p}<.01$ ), but the control group showed a more dramatic decrease than either the septal or the hippocampal group ( $t$ tests, $\mathrm{p}<.01$ ). The septal and hippocampal groups did not differ (t test, $\mathrm{p}>.05$ ). Thus, both the septal and hippocampal operates showed a significant and approximately equal deficit in taste aversion learning under the conditions of this experiment. The extent of the hippocampal lesions is shown in Figure 2. Septal lesions were very similar to those of Experiment 1.

\section{Discussion}

There are a number of procedural changes that might account for the change from total suppression of fluid consumption on the baitshyness tests of Experiment 1 to the milder aversion demonstrated by all groups in Experiment 2. One important change is the switch to a preferred CS, $15 \%$ sucrose. While all groups showed a drop in fluid consumption (neophobia) when first confronted with the LiCl CS

Table 2

Mean Volume (in Milliliters) of Fluid Consumed in 15-min Period by Rats with Septal or Hippocampal Lesions

\begin{tabular}{|c|c|c|c|c|c|c|c|}
\hline \multirow[b]{2}{*}{ Group } & \multirow[b]{2}{*}{$\mathrm{N}$} & \multicolumn{2}{|c|}{ Days 1-3 $\left(\mathrm{H}_{2} \mathrm{O}\right)$} & \multicolumn{2}{|c|}{ Day 4 (Sucrose) } & \multicolumn{2}{|c|}{ Day 7 (Sucrose) } \\
\hline & & Mean & Range & Mean & Range & Mean & Range \\
\hline $\begin{array}{l}\text { Control } \\
\text { Hippocampal } \\
\text { Septal }\end{array}$ & $\begin{array}{r}11 \\
8 \\
7\end{array}$ & $\begin{array}{l}13.1 \\
14.7 \\
15.3\end{array}$ & $\begin{array}{l}5.8-20.2 \\
8.6-20.8 \\
9.6-17.5\end{array}$ & $\begin{array}{l}19.8 \\
23.3 \\
25.2\end{array}$ & $\begin{array}{r}7-26 \\
18-31 \\
18-32\end{array}$ & $\begin{array}{r}9.3 \\
14.4 \\
16.6\end{array}$ & $\begin{array}{l}2-15 \\
6-22 \\
7-26\end{array}$ \\
\hline
\end{tabular}



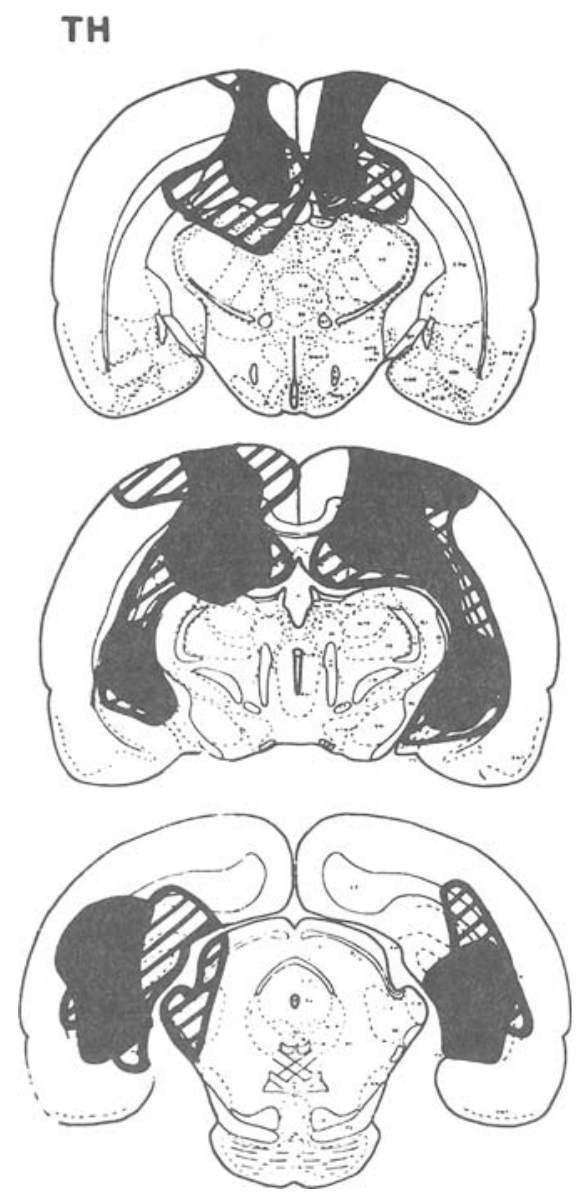

Figure 2. Reconstruction of the smallest (black) and largest (diagonal lines) lesion in the total hippocampal (TH) lesion group of Experiment 2.

of Experiment 1, all groups showed an increased fluid consumption (preference) when first exposed to the sucrose CS of Experiment 2. Also, the route of administration of the US and the interstimulus delay differed dramatically in the two experiments. The self-administration technique of the first experiment resulted in an intragastric delivery of the lithium with a minimal interstimulus delay. In the second experiment there was a 15 -min interstimulus delay before the IP injection of the US. Finally, the amount of lithium ingested by most rats in Experiment 1 (approximately $30 \mathrm{ml} / \mathrm{kg}$ of .12-M LiCl) was approximately six times greater than that injected in Experiment $2(2 \mathrm{ml} / \mathrm{kg}$ of . $3-\mathrm{M} \mathrm{LiCl})$.

\section{GENERAL DISCUSSION}

The results of these experiments, considered together, show that septal or hippocampal lesions can disrupt the learning or expression of conditioned taste aversions under certain conditions. However, the septo-hippocampal system is apparently not critically involved in taste aversion learning in a general sense, since strong aversions can be conditioned even after large lesions that completely destroy the septum, the dorsal hippocampus, or the fibers interconnecting septum and hippocampus (Experiment 1). These findings, considered together with reports of locomotor passive avoidance impairments without disruption of baitshyness (Hobbs et al., 1974; McGowan et al., 1969; McGowan et al., 1972), might be taken as support for the idea that taste aversion learning is mediated by a different neural system from that involved in the association of exteroceptive cues and reinforcers. That is, the septum and hippocampus might represent critical elements in a neural system subserving locomotor passive avoidance learning or performance while having little or nothing to do with baitshyness acquisition or expression. This remains a logical possibility. However, the extant data, including the results of this study, are equally supportive of the idea that conditioned taste aversions are simply more difficult to disrupt with neural interventions than are some other forms of associative learning (Best \& Orr, 1973).

It should be noted that the reported dissociation between taste aversion and locomotor passive avoidance after hippocampal lesions is incomplete. For example, Best and Orr (1973) found that dorsal hippocampal lesions disrupted both passive avoidance learning and taste aversion acquisition, and Miller et al. (1975) reported that total hippocampal lesions impaired both passive avoidance learning and taste aversion extinction. Both of these studies point out the difficulty of equating stimulus parameters when comparing the results of shock avoidance and taste aversion learning. Such parameters are important determinants of the strength of conditioning and hence of the appearance of lesion-induced deficits.

In general, hippocampal lesions have produced taste aversion deficits in those studies that have used weak conditioning paradigms (Best \& Orr, 1973; Krane et al., 1976; McFarland et al., in press; Miller et al., 1971; Experiment 2, present study), whereas no hippocampal lesion effects have been obtained in those studies that achieved the strongest taste aversion conditioning (Kolb et al., 1977; McGowan et al., 1972; Murphy \& Brown, 1974; Experiment 1, present study). Taste aversion conditioning can be weakened in several ways, including preexposure to the CS, the use of a preferred CS, interpolation of a familiar taste stimulus between presentation of a novel CS and the US, the use of a low-intensity US, or combinations of the above. In the present study, deficits in taste aversion acquisition were seen after either septal or hippocampal lesions when a preferred CS (sucrose) was coupled with a weak LiCl US, but no lesion-induced deficit was obtained when a non- 
preferred $\mathrm{CS}(\mathrm{LiCl})$ was paired with a strong $\mathrm{LiCl}$ US. The most dramatic demonstration of a baitshyness deficit to date was obtained when a latent inhibition paradigm (CS preexposure) was coupled with a preferred saccharine CS (Miller et al., 1971). In this situation, animals with large hippocampal lesions demonstrated complete disruption of taste aversion learning. Finally, Miller et al. (1975) showed that it is possible to demonstrate hippocampal lesion effects under prolonged extinction even when a strong conditioning paradigm precluded the appearance of an initial deficit in taste aversion acquisition.

All of these results are consistent with traditional theories that ascribe to the hippocampus a role in the inhibition of prepotent response tendencies (Douglas, 1967) or in the inhibition of attention to previously nonreinforced stimuli (Kimble, 1968). That is, hippocampal lesions seem to affect baitshyness under the same conditions (latent inhibition, extinction, avoidance of preferred CS) as they affect associations of exteroceptive cues and reinforcers. Thus, it is not yet possible to state that the association of taste cues with interoceptive events depends on different neural circuits than the association of exteroceptive cues and events. Previous authors (Best \& Orr, 1973; Miller et al., 1975) have noted that a demonstration of lesion-induced disruption of taste aversion in the absence of a disruption of shock-motivated avoidance is needed before the issue of separate neural mechanisms can be resolved. In fact, the requirements are even more stringent. Because both taste aversion and shock-motivated avoidance are strongly influenced by stimulus intensity, a double dissociation between lesion locus and behavioral effect is required. That is, if separate neural mechanisms are truly involved in the two types of learning, it should be possible to demonstrate, within the same experiment, a disruption of taste aversion and not shock-motivated avoidance with one lesion and a disruption of shockmotivated avoidance but not taste aversion with another lesion. Until such an effect is demonstrated, the hypothesis of separate neural control remains only an intriguing possibility.

\section{REFERENCES}

Best, P. J., \& ORR, J. Effects of hippocampal lesions on passive avoidance and taste aversion conditioning. Physiology \& Behavior, 1973, 10, 193-196.

Douglas, R. J. The hippocampus and behavior. Psychological Bulletin, 1967, 67, 416-422.
Garcia, J., \& Koelling, R. A. Relation of cue to consequence in avoidance learning. Psychonomic Science, 1966, 4, 123-124.

Hinde, R. A. (Ed.). Constraints on learning. New York: Academic Press, 1973.

Hobbs, S. H., Elkins, R. L., \& Peacock, L. J. Taste-aversion conditioning in rats with septal lesions. Behavioral Biology, 1974, 11, 239-245.

Kimble, D. P. Hippocampus and internal inhibition. Psychological Bulletin, 1968, 70, 285-295.

Kolb, B., Nonneman, A. J., \& Abplanalp, P. Studies on the neural mechanisms of bait-shyness in rats. Bulletin of the Psychonomic Society, 1977, 10, 389-392.

Krane, R., Sinnamon, H., \& Thomas, G. Conditioned taste aversions and neophobia in rats with hippocampal lesions. Journal of Comparative and Physiological Psychology, 1976, 90, 680-693.

McFarland, D. J., Kostas, J., \& Drew, W. G. Dorsal hippocampal lesions: Effects of pre-conditioning CS exposure on flavor aversion. Behavioral Biology, in press.

McGowan, B. K., Garcia, J., Ervin, F. R., \& Schwartz, J. Effects of septal lesions on bait-shyness in the rat. Physiology \& Behavior, 1969, 4, 907-909.

McGowan, B. K., Hankins, W. G., \& Garcia, J. Limbic lesions and control of the internal and external environment. Behavioral Biology, 1972, 7, 841-852.

Miller, C. R., Elkins, R. L., Fraser, J., Peacock, L. J., \& Hoвbs, S. H. Taste aversion and passive avoidance in rats with hippocampal lesions. Physiological Psychology, 1975, 3, 123-126.

Miller, C. R., Elkins, R. L., \& Peacock, L. J. Disruption of radiation-induced preference shift by hippocampal lesions. Physiology \& Behavior, 1971, 6, 283-285.

Murphy, L. R., \& Brown, T. S. Hippocampal lesions and learned taste aversion. Physiological Psychology, 1974, 2, 60-64.

REVUSKY, S., \& GoRRY, T. Flavor aversions produced by contingent drug injection: Relative effectiveness of apomorphine, emetine, and lithium. Behavioral Research and Therapy, 1973, 11. 403-409.

Rozin, P., \& Kalat, J. W. Specific hungers and poison avoidance as adaptive specializations of learning. Psychological Review, 1971, 78, 459-486.

Seligman, M. E. P., \& HAger, J. L. (Eds.) Biological boundaries of learning. New York: Appleton-Century-Crofts, 1972.

SkInNer, J. E. Neuroscience: A laboratory manual. Philadelphia: Saunders, 1971.

ThomkA, M. L., \& Brown, T. S. The effect of hippocampal lesions on the development and extinction of a learned taste aversion for a novel food. Physiological Psychology, 1975, 3, 281-284.

\section{NOTE}

1. McFarland, Kostas, and Drew (in press) subsequently reported deficient taste aversion learning with a walnut extract CS and $\mathrm{Li}_{2} \mathrm{CO}_{3} \mathrm{US}$, but only when the animals had been preexposed to the CS.

(Received for publication November 16, 1977; revision accepted February 22, 1978.) 